\title{
The principle of relative locality
}

\author{
Giovanni Amelino-Camelia ${ }^{a}$, Laurent Freidel $^{c}$, Jerzy Kowalski-Glikman ${ }^{b}$, Lee Smolin ${ }^{c}$ \\ ${ }^{a}$ Dipartimento di Fisica, Università "La Sapienza" and Sez. Roma1 INFN, P.le A. Moro 2, 00185 Roma, Italy \\ ${ }^{b}$ Institute for Theoretical Physics, University of Wroclaw, Pl. Maxa Borna 9, 50-204 Wroclaw, Poland \\ ${ }^{c}$ Perimeter Institute for Theoretical Physics, \\ 31 Caroline Street North, Waterloo, Ontario N2J 2Y5, Canada \\ (Dated: February 1, 2011)
}

\begin{abstract}
We propose a deepening of the relativity principle according to which the invariant arena for non-quantum physics is a phase space rather than spacetime. Descriptions of particles propagating and interacting in spacetimes are constructed by observers, but different observers, separated from each other by translations, construct different spacetime projections from the invariant phase space. Nonetheless, all observers agree that interactions are local in the spacetime coordinates constructed by observers local to them.

This framework, in which absolute locality is replaced by relative locality, results from deforming momentum space, just as the passage from absolute to relative simultaneity results from deforming the linear addition of velocities. Different aspects of momentum space geometry, such as its curvature, torsion and non-metricity, are reflected in different kinds of deformations of the energy-momentum conservation laws. These are in principle all measurable by appropriate experiments. We also discuss a natural set of physical hypotheses which singles out the cases of momentum space with a metric compatible connection and constant curvature.
\end{abstract}

\section{INTRODUCTION}

How do we know we live in a spacetime? And if so how do we know we all share the same spacetime? These are the fundamental questions we are investigating in this note.

We local observers do not directly observe any events macroscopically displaced from our measuring instruments. As naive observers looking out at the world, and no less as particle physicists and astronomers, we are basically "calorimeters" with clocks. Our most fundamental measurements are the energies and angles of the quanta we emit or absorb, and the times of those events. Judging by what we observe, we live in momentum space, not in spacetime.

The idea that we live in a spacetime is constructed by inference from our measurements of momenta and energy. This was vividly illustrated by Einstein's procedure to give spacetime coordinates to distant events by exchanges of light signals [1]. When we use Einstein's procedure we take into account the time it takes the photons to travel back and forth but we throw away information about their energy, resulting in a projection into spacetime. When we do this we presume that the same spacetime is reconstructed by exchanges of light signals of different frequencies. We are also used to assuming that different local observers, distant from each other, reconstruct the same spacetime by measurements of photons they send and receive.

But why should the information about the energy of the photons we use to probe spacetime be inessential? Might that just be a low energy approximation? And why should we presume that we construct the same spacetime from our observations as observers a cosmological distance from us?

In this paper we show that absolute locality, which postulates that all observers live in the same space time, is equivalent to the assumption that momentum space is a linear manifold. This corresponds to an idealization in which we throw away the information about the energy of the quanta we use to probe spacetime and it can be transcended in a simple and powerful generalization of special relativistic physics which is motivated by general considerations of the unification of gravity with quantum physics. In this work we link the notion of locality with assumptions made about the geometry of momentum space. We propose a new framework in which we can relax in a controlled manner the concept of absolute locality by linking this to a new understanding of the geometry of momentum space. In this framework there is no notion of absolute locality, different observers see different spacetimes, and the spacetimes they observe are energy and momentum dependent. Locality, a coincidence of events, becomes relative: coincidences of events are still objective for all local observers, but they are not in general manifest in the spacetime coordinates constructed by distant observers.

One way to motivate this new physical framework is by thinking about the symmetry of the vacuum. The most basic question that can be asked of any physical system is what is the symmetry of the ground state that governs its low lying excitations. This is no less true of spacetime itself, moreover in general relativity, and presumably in any description of the quantum dynamics of spacetime, the symmetry of the ground state is dynamically determined. We also expect that the classical spacetime geometry of general relativity is a semiclassical approximation to a more fundamental quantum geometry. In this paper we show how simple physical assumptions about the geometry of momentum space may control the departure of the spacetime description from the classical one.

We will first restrict attention to an approximation in which $\hbar$ and $G_{N e w t o n}$ both may be neglected while their ratio

$$
\sqrt{\frac{\hbar}{G_{\text {Newton }}}}=m_{p}
$$

is held fixed ${ }^{1}$. In this approximation quantum and gravita-

\footnotetext{
${ }^{1}$ We work in units in which $c=1$.
} 
tional effects may both be neglected, but there may be new phenomena on scales of momentum or energy given by $m_{p}$. At the same time, because $l_{p}=\sqrt{\hbar G_{\text {Newton }}} \rightarrow 0$ we expect no features of quantum spacetime geometry to be relevant.

Since our approximation gives us an energy scale, but not a length scale, we will begin by presuming that momentum space is more fundamental than spacetime. This is in accord with the operational point of view we mentioned in the opening paragraph. So we begin in momentum space by asking how it may be deformed in a way that is measured by a scale $m_{p}$. Once that is established we will derive the properties of spacetime from dynamics formulated in momentum space. For convenience we work in first in the limit just described, after which we will briefly turn on $\hbar$.

By following this logic below, we will find that physics may be governed by a novel principle, which we call the Principle of Relative Locality. This states that,

Physics takes place in phase space and there is no invariant global projection that gives a description of processes in spacetime. From their measurements local observers can construct descriptions of particles moving and interacting in a spacetime, but different observers construct different spacetimes, which are observer-dependent slices of phase space.

In the next section we introduce an operational approach to the geometry of momentum space, which we build on in section III to give a dynamics of particles on a curved momentum space. We see how a modified version of spacetime geometry is emergent from the dynamics which is formulated on momentum space. In these sections we consider a general momentum space geometry, which illuminates a variety of new phenomena that might be experimentally probed corresponding to the curvature, torsion and non-metricity of momentum space. However, one advantage of this approach is that with a few reasonable physical principles the geometry of momentum space can be reduced to three choices, depending on the sign of a parameter. As we show in section IV, this gives this framework both great elegance and experimental specificity. In section $\mathrm{V}$ we make some preliminary observations as to how the geometry of momentum space may be probed experimentally, after which we conclude.

\section{AN OPERATIONAL APPROACH TO THE GEOMETRY OF MOMENTUM SPACE}

We take an operational point of view in which we describe physics from the point of view of a local observer who is equipped with devices to measure the energy and momenta of elementary particles in her vicinity. The observer also has a clock that measures local proper time. We construct the ge- ometry of momentum space ${ }^{2} \mathcal{P}$ from measurements made of the dynamics of interacting particles. We assume that to each choice of calorimeter and other instruments carried by our observer there is a preferred coordinate on momentum space, $k_{a}$. But we also assume that the dynamics can be expressed covariantly in terms of geometry of $\mathcal{P}$ and do not depend on the choice of calorimeter's coordinates. We note that the $k_{a}$ measure the energy and momenta of excitations above the ground state, hence the origin of momentum space, $k_{a}=0$, is physically well defined.

Our local observer can make two kinds of measurements. One type of measurement can be done only with a single particle and it defines, as we will see, a metric on momentum space. The other type of measurement involve multi particles and defines a connection. A key mathematical idea underlying our construction is that a connection on a manifold can be determined by an algebra [2], in the present case this will be an algebra that determines how momenta combine when particles interact.

\section{A. The metric geometry of momentum space}

First we describe the metric geometry. Our local observer can measure the rest energy or relativistic mass of a particle which is a function of the four momenta. She can also measure the kinetic energy $K$ of a particle of mass $m$ moving with respect to her, but local to her. We postulate that these measurements determine the metric geometry of momentum space. We interpret the mass as the geodesic distance from the origin, this gives the dispersion relation

$$
D^{2}(p) \equiv D^{2}(p, 0)=m^{2} .
$$

The measurement of kinetic energy defines the geodesic distance between a particle $p$ at rest and a particle $p^{\prime}$ of identical mass and kinetic energy $K$, that is $D(p)=D\left(p^{\prime}\right)=m$ and

$$
D^{2}\left(p, p^{\prime}\right)=-2 m K \text {. }
$$

The minus sign express the fact that the geometry of momentum space is Lorentzian. From these measurements one can reconstruct a metric on $P^{3}$

$$
d k^{2}=h^{a b}(k) d k_{a} d k_{b}
$$

\section{B. The algebra of interactions}

Now we describe the construction of the connection on momentum space. This is determined by processes in which

\footnotetext{
${ }^{2} \mathrm{By}$ which we mean the space of relativistic four-momenta denoted $p_{a}$ with $a=0,1,2,3$.

${ }^{3}$ In the standard case of physics in Minkowski spacetime, $h^{a b}$ is the dual Minkowski metric and $\mathcal{K}_{a}(k)=\sum_{I} k_{a}^{I}$. A scale $m_{p}$ may be introduced by deforming the geometry of $\mathcal{P}$ so that it is curved. The correspondence principle (to be introduced below) assures that we recover the standard flat geometry of $\mathcal{P}$ in the limit $m_{p} \rightarrow \infty$.
} 
$n$ particles interact, $n_{\text {in }}$ incoming and $n_{\text {out }}$ outgoing, with $n=n_{\text {in }}+n_{\text {out }}$. This proceeds by the construction of an algebra, which then determines the connection.

Associated to each interaction there must be a combination rule for momentum, which will in general be non-linear. We denote this rule for two particles by

$$
(p, q) \rightarrow p_{a}^{\prime}=(p \oplus q)_{a}
$$

Hence the momentum space $\mathcal{P}$ has the structure of an algebra defined by the product rule $\oplus$. We assume that more complicated processes are built up by iterations of this product - but to begin with we assume neither linearity, nor commutativity nor associativity.

We will also need an operation that turns outgoing momenta into incoming momenta. This is denoted, $p \rightarrow \ominus p$ and it satisfies $^{4}$

$$
(\ominus p) \oplus p=0
$$

Then we have the conservation law of energy and momentum for any process, giving, for each type of interaction, four functions on $\mathcal{P}^{n}$, depending on momenta of interacting particles, which vanish

$$
\mathcal{K}_{a}\left(k^{I}\right)=0
$$

For example, for a process with three incoming momenta $p_{a}$, $q_{a}$, and $k_{a}$ one might make the choice

$$
\mathcal{K}_{a}(p, q, k)=p_{a} \oplus\left(q_{a} \oplus k_{a}\right)=0
$$

\section{From the algebra of interactions to the connection on momentum space}

Corresponding to the algebra of combinations of momenta there is a connection on $\mathcal{P}$. The geometry of momentum space is studied in detail in [2], but the basics are as follows. The algebra of the combination rule determines a connection on $P$ by

$$
\left.\frac{\partial}{\partial p_{a}} \frac{\partial}{\partial q_{b}}(p \oplus q)_{c}\right|_{q, p=o}=-\Gamma_{c}^{a b}(0)
$$

The torsion of $\Gamma_{a}^{b c}$ is a measure of the asymmetric part of the combination rule

$$
-\frac{\partial}{\partial p_{a}} \frac{\partial}{\partial q_{b}}\left((p \oplus q)_{c}-(p \oplus q)_{c}\right)_{q, p=o}=T_{c}^{a b}(0)
$$

Similarly the curvature of $\mathcal{P}$ is a measure of the lack of associativity of the combination rule

$$
\left.2 \frac{\partial}{\partial p_{[a}} \frac{\partial}{\left.\partial q_{b}\right]} \frac{\partial}{\partial k_{c}}((p \oplus q) \oplus k-p \oplus(q \oplus k))_{d}\right|_{q, p, k=o}=R_{d}^{a b c}(0)
$$

\footnotetext{
${ }^{4}$ And more generally $(\ominus p) \oplus(p \oplus k)=k$, where $\ominus$ is a left inverse.
}

where the bracket denote the anti-symmetrisation.

We note that there is no physical reason to expect a combination rule for momentum to be associative, once it is nonlinear. Indeed, the lack of associativity means there is a physical distinction between the two processes of Figure 1, which is equivalent to saying there is a definite microscopic causal structure. That is, causal structure of the physics maps to nonassociativity of the combination rule for momentum which in turn maps to curvature of momentum space. The curvature of momentum space makes microscopic causal orders distinguishable, and hence meaningful. This gives rise to proposals to measure the curvature of momentum space which we will discuss below.

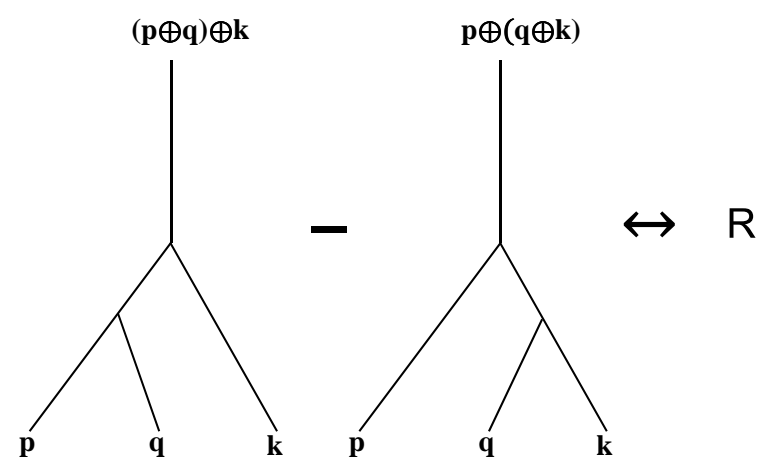

FIG. 1: Curvature of the connection on momentum space produces non-associativity of composition rule.

To determine the connection, torsion and curvature away from the origin of momentum space we have to consider translating in momentum space, ie we can denote

$$
p \oplus_{k} q=k \oplus((\ominus k \oplus p) \oplus(\ominus k \oplus q))
$$

the identity for this product is at $0_{k}=k$. Then

$$
\left.\frac{\partial}{\partial p_{a}} \frac{\partial}{\partial q_{b}}\left(p \oplus_{k} q\right)_{c}\right|_{q, p=k}=-\Gamma_{c}^{a b}(k)
$$

Thus, the action of adding an infinitesimal momentum $d q_{a}$ from particle $J$ to a finite momentum $p_{a}$ of particle $I$ defines a parallel transport on $\mathcal{P}$.

$$
p_{a} \oplus d q_{a}=p_{a}+d q_{b} \tau_{a}^{b}(p)
$$

where $\tau(p)$ is the parallel transport operation from the identity to $p$. It can be expanded around $p=0$

$$
\tau_{a}^{b}(p)=\delta_{a}^{b}-\Gamma_{a}^{b c} p_{c}-\Gamma_{a}^{b c d} p_{c} p_{d}+\cdots
$$

with

$$
\Gamma_{a}^{b c d}=\partial_{p}^{d} \Gamma_{a}^{b c}-\Gamma_{i}^{d b} \Gamma_{a}^{i c}-\Gamma_{i}^{d c} \Gamma_{a}^{b i}
$$


The corresponding conservation law thus has the form to second order

$$
\mathcal{K}_{a}(k)=\sum_{I} k_{a}^{I}-\sum_{J \in \mathcal{I}(I)} C_{I, J} \Gamma_{a}^{b c} k_{b}^{J} k_{c}^{I}+\ldots
$$

where $\mathcal{I}(I)$ is the set of particles that interact with the I's one and $C_{I, J}$ are coefficients that depend on the form of the conservation law.

We will shortly study the consequences of curvature and torsion on momentum space for the dynamics of particles. We will see that the meaning of the curvature of momentum space is that it implies a limitation of the usefulness of the notion that processes happen in an invariant spacetime, rather than in phase space. The hypothesis of a shared, invariant spacetime, in which all observers agree on the locality of distant interactions, turns out to be a direct consequence of the linearity of the usual conservation laws of energy and momentum. When we deform the conservation laws by making them non-linear, this gives rise to relative locality.

\section{THE EMERGENCE OF SPACETIME FROM THE DYNAMICS OF PARTICLES}

We take the point of view that spacetime is an auxiliary concept which emerges when we seek to define dynamics in momentum space. If we take the momenta of elementary particles to be primary, they themselves need momenta, so that a canonical dynamics can be formulated. The momenta of the momenta are quantities $x_{I}^{a}$ that live in the cotangent space of $\mathcal{P}^{n}$ at a point $k_{a}^{I}$.

\section{A. Variational principle}

Given these we can define the free particle dynamics by

$$
S_{\text {free }}^{I}=\int d s\left(x_{J}^{a} \dot{k}_{a}^{J}+\mathcal{N}_{J} C^{J}(k)\right)
$$

where $s$ is an arbitrary time parameter and $\mathcal{N}_{J}$ is the Lagrange multiplier imposing the mass shell condition

$$
C^{J}(k) \equiv D^{2}(k)-m_{J}^{2}
$$

We emphasize that the contraction $x_{J}^{a} \dot{k}_{a}^{J}$ does not involve a metric, and the dynamics is otherwise given by constraints which are functions only of coordinates on $\mathcal{P}$ and depend only the geometry of $\mathcal{P}$. This leads to the Poisson brackets,

$$
\left\{x_{I}^{a}, k_{b}^{J}\right\}=\delta_{b}^{a} \delta_{I}^{J}
$$

We then have a phase space, $\Gamma$ of a single particle, which is the cotangent bundle of $\mathcal{P}$. We note that there is neither an invariant projection from $\Gamma$ to a spacetime, $\mathcal{M}$ nor is there defined any invariant spacetime metric. Yet this structure is sufficient to describe the dynamics of free particles. The fact that there is no invariant projection to a spacetime is related to the non linearity of momentum space. Indeed under a non linear redefinition $p \rightarrow F(p)$ the conjugated coordinates is given by $x \rightarrow(\partial p / \partial F) x$, so the new canonical coordinate appears to be momentum space dependent. This is this mixing between "spacetime" and momentum space that is the basis of the relative locality. We can call the $x_{J}^{a}$ Hamiltonian spacetime coordinates because they are defined as being canonically conjugate to coordinates on momentum space.

Note that we do not need a spacetime or spacetime metric to describe how these particles interact. If we consider the process with $n$ interacting particles we want to impose conservation of the non-linear quantities, $\mathcal{K}_{a}$. We do this by introducing a lagrange multiplier to guarantee conservation (7). The action is

$$
S^{\text {total }}=\sum_{J} S_{\text {free }}^{J}+S^{\text {int }}
$$

where for the incoming particles

$$
S_{\text {free }}^{J}=\int_{-\infty}^{0} d s\left(x_{J}^{a} \dot{k}_{a}^{J}+\mathcal{N}_{J} C^{J}(k)\right)
$$

while for the outgoing particles

$$
S_{\text {free }}^{J}=\int_{0}^{\infty} d s\left(x_{J}^{a} \dot{k}_{a}^{J}+\mathcal{N}_{I} C^{J}(k)\right)
$$

The interaction contribution to the action is simply a lagrange multiplier times the conservation law (7).

$$
S^{i n t}=\mathcal{K}(k(o))_{a} z^{a}
$$

We have set the interaction to take place at affine parameter $s=0$ for each of the particles. At this point $z^{a}$ can be just considered to be a lagrange multiplier to enforce the conservation of momentum (7) at the interaction where for each particle $s=0$.

We vary the total action. After an integration by parts in each of the free actions we have

$$
\begin{gathered}
\delta S^{\text {total }}= \\
\sum_{J} \int_{s_{1}}^{s_{2}}\left(\delta x_{J}^{a} \dot{k}_{a}^{J}-\delta k_{a}^{J}\left[\dot{x}_{J}^{a}-\mathcal{N}_{J} \frac{\delta C^{J}}{\delta k_{a}^{J}}\right]+\delta \mathcal{N}_{I} C^{J}(k)\right)+\mathcal{R}
\end{gathered}
$$

Here $\mathcal{R}$ contains both the result of varying $S^{\text {int }}$ and the boundary terms from the integration by parts. $s_{1,2}$ are $0, \infty,-\infty$ depending on whether the term is incoming or outgoing. Before examining the boundary terms we confirm we have the desired free parts of the equations of motion

$$
\begin{aligned}
\dot{k}_{a}^{J} & =0 \\
\dot{x}_{J}^{a} & =\mathcal{N}_{J} \frac{\delta C^{J}}{\delta k_{a}^{J}} \\
C^{J}(k) & =0
\end{aligned}
$$

We fix $\delta k_{a}^{J}=0$ at $s= \pm \infty$ and examine the remainder of the variation

$$
\mathcal{R}=\mathcal{K}(k)_{a} \delta z^{a}-\left(x_{J}^{a}(0)-z^{b} \frac{\delta \mathcal{L}_{b}}{\delta k_{a}^{J}}\right) \delta k_{a}^{J}
$$


Here $x_{J}^{a}$ and $k_{a}^{J}$ are taken for each particle at the parameter time $s=0$. This has to vanish if the variational principle is to have solutions. From the vanishing of the coefficient of $\delta z^{a}$ we get the four conservation laws of the interaction (7). From the vanishing of the coefficient of $\delta k_{a}^{J}$ we find $4 n$ conditions which hold at the interaction

$$
x_{J}^{a}(0)=z^{b} \frac{\delta \mathcal{L}_{b}}{\delta k_{a}^{J}}
$$

Using [17] this gives conditions

$$
x_{J}^{a}(0)=z^{a}-z^{b} \sum_{L \in \mathcal{I}(J)} C_{J, L} \Gamma_{b}^{a c} k_{c}^{L}+\ldots
$$

This tells us that to leading order, in which we ignore the curvature of momentum space, all of the worldlines involved in the interaction meet at a single spacetime event, $z^{a}$. The choice of $z^{a}$ is not constrained and cannot be, for its variation gives the conservation laws (7). Thus, we have recovered the usual notion that interactions of particles take place at single events in spacetime from the conservation of energy and momentum. This is good because in quantum field theory conservation implies locality, and it is good to have a formulation of classical interactions where this is also the case.

However when we include terms proportional to $z^{a}$, which is to say when the observer is not at the interaction event, we see that the relationship between conservation of energy and momentum and locality of interactions is realized a bit more subtly. The interaction takes place when the condition 293 is satisfied, that is at $n$ separate events, separated from $z^{a}$ by intervals

$$
\Delta x_{J}^{a}(0)=-z^{b} \sum_{L \in \mathcal{J}(J)} C_{J, L} \Gamma_{b}^{a c} k_{c}^{L}+\ldots
$$

These relations (29), (30) illustrate concisely the relativity of locality. For some fortunate observers the interaction takes place at the origin of their coordinates, so that $z^{a}=x_{J}^{a}(0)=0$ in which case the interaction is observed to be local. Any other observer, translated with respect to these, has a non-vanishing $z^{a}$ and hence sees the interaction to take place at a distant set of events. These are centered around $z^{a}$ but are not precisely at the same values of the coordinates. That is the coordinates of particles involved in an interaction removed from the origin of the observer by a vector $z^{a}$ are spread over a region of order

$$
\Delta x \approx|z||\Gamma| k
$$

The relationship (28) possess a very nice mathematical meaning too. Since the momentum space is in general curved the proper way to define the conjugate coordinates is as elements of the cotangent bundle of $\mathcal{P}$. The cotangent space based at $p^{I}$ and the cotangent space based at 0 are different spaces in the general curved case. This expresses mathematically the relativity of locality. The hamiltonian particle coordinate $x_{I}$ represent an element of $T_{p^{I}}^{*} \mathcal{P}$ while the interaction coordinate being dual to the conservation law represent an element of $T_{0}^{*} \mathcal{P}$. (28) represent a relation between these two spaces and remarkably it can be shown [2] that indeed the coefficient $\partial \mathcal{K} / \partial k$ evaluated when $\mathcal{K}=0$ is the parallel transport operator, more precisely

$$
\left.\partial_{p}^{b}(k \oplus p)_{a}\right|_{p=\ominus k}=\left(\tau(k)^{-1}\right)_{a}^{b}
$$

where $\tau(k)$ is the parallel transport operator of vectors from 0 to $k$ introduced earlier therefore $\tau(k)^{-1}$ is the parallel transport operator of covectors from 0 to $k$.

\section{B. The physical meaning of relative locality}

Is this a real, physical non-locality or a new kind of coordinate artifact? It is straightforward to see that it is the latter, because the $\Delta x_{J}^{a}(0)$ can be made to vanish by making a translation to the coordinates of another observer. In a canonical formulation, translations are generated by the laws of conservation of energy and momentum, (7). Given any local observable in phase space $O$ observed by a local observer, Alice, we can construct the observable as seen in coordinates constructed by another observer, Bob, distant from Alice, by a translation labeled by parameters $b^{a}$.

$$
\delta_{b} O=b^{b}\left\{\mathcal{K}_{b}, O\right\}
$$

Since momentum space is curved, and $\mathscr{K}_{b}$ is non-linear, it follows that the "spacetime coordinates" $x_{J}^{a}$ of a particle translate in a way that is dependent on the energy and momenta of the particles it interacts with, $x_{J}^{a} \rightarrow x_{J}^{\prime a}(0)=x_{J}^{a}(0)+\delta_{b} x_{J}^{a}(0)$ where

$$
\delta_{b} x_{J}^{a}(0)=b^{b}\left\{\mathcal{K}_{b}, x_{J}^{a}\right\}=-b^{a}+b^{b} \sum_{L \in \mathcal{I}(J)} C_{J, L} \Gamma_{b}^{a c} k_{c}^{L}+\ldots
$$

This is a manifestation of the relativity of locality, ie local spacetime coordinates for one observer mix up with energy and momenta on translation to the coordinates of a distant observer.

This mixing under translations effect also entirely accounts for the separation of an interaction into apparently distinct events, because with $b^{b}=-z^{b}$, we see that $\Delta x_{J}^{a}$ of 28 ] is equal to $\delta_{b} x_{J}^{a}(0)$ of (34). Thus, the observer whose new coordinates we have translated to observes a single interation taking place at $x_{J}^{a} \rightarrow x_{J}^{\prime a}(0)=0$.

Thus, if I am a local observer and see an interaction to take place via a collision at my origin of coordinates, a distant observer will generally see it in their coordinates as spread out in space-time by (28). And vice versa. There is not a physical non-locality, as all momentum conserving interactions are seen as happening at a single spacetime event by some family of observers, who are local to the interaction. But it becomes impossible to localize distant interactions in an absolute manner. Furthermore, all observers related by a translation agree about the momenta of the particles in the interaction, because under translations $33, \delta_{b} k_{a}^{I}=0$.

Note that if the curvature and torsion vanish there is no mixing of spacetime coordinates with momenta under translations, so there is an invariant definition of spacetime. Thus, the flatness of momentum space is responsible for the notion of an absolute spacetime, just as the additivity of velocity allows Newtonian physics to have an absolute time. 
Note also that the translations of spacetime coordinates define sections $S$ on $\Gamma$, which extend from the origin of local coordinates. These tell us how to translate events at the origin of the coordinates of an observer to coordinates measured by a distant observer. These sections provide local and energy dependent definitions of space-time, relative to observers and energy scales. These sections are defined by Hamiltonian vector fields on $\Gamma$ which are defined acting on functions $f$ on $\Gamma$ by

$$
v_{b} f=\left\{b^{a} \mathcal{K}_{a}, f\right\}=b^{a} \sum_{J}\left(1-\sum_{K \in \mathcal{I}(J)} C_{J, K} \Gamma_{a}^{b c} k_{c}^{K}+\ldots\right) \frac{\partial f}{\partial x_{J}^{a}}
$$

We can check that these commute and hence define submanifolds of $\Gamma$. We can define an inverse metric on the sections $\mathcal{S}$ defined by

$$
g^{a b}(x, k)=g\left(d x^{a}, d x^{b}\right)=h^{c d}(k)\left\{\mathcal{K}_{c}, x^{a}\right\}\left\{\mathcal{K}_{d}, x^{b}\right\}
$$

We note that this metric on the sections $\mathcal{S}$ is momentum dependent. Thus, we arrive at a description of the geometry of spacetime which is energy dependent. This metric is in fact just the fiber metric at the point $k$ where the fiber is the cotangent plane at this point ${ }^{5}$.

But note that we can take all the $k_{a}^{I}=0$ in which case $g^{a b}(x, k=0)=\eta^{c d}$ is the Minkowski metric. So observers who probe spacetime with zero momentum probes will see Minkowski spacetime. However, the coordinates of this invariant zero momentum section are non-commutative.

$$
\left\{z^{a}, z^{b}\right\} \neq 0
$$

Indeed, if one wants to describe the spacetime as probed by the zero momentum probes this means that we desire to model the spacetime as the cotangent space of the origin. In order to achieve this we need to parallel transport the event at $p$ back to events at 0 . This means that we interpret the coordinates $z$ as being covariant covector fields defined by

$$
z^{a}=\tau(p)_{b}^{a} x_{p}^{b}
$$

where $x_{p}$ is the coordinate dual to $p$ with respect to the poisson bracket and living in the cotangent space at $p$. The Poisson commutator can now be evaluated, it is related to the Lie bracket of the covariantly constant vector field on $\mathcal{P}$ and its expansion is given by

$$
\begin{aligned}
\left\{z^{a}, z^{b}\right\} & =\left(\tau_{\bar{a}}^{a} \partial^{\bar{a}} \tau_{\bar{b}}^{b}-\tau_{\bar{a}}^{b} \partial^{\bar{a}} \tau_{\bar{b}}^{a}\right) x^{\bar{b}} \\
& =T_{d}^{a b} z^{d}+R^{a b c}{ }_{d} p_{c} z^{d}+\cdots
\end{aligned}
$$

where we have expanded around $p=0$ in the second equality.

\footnotetext{
5 We are usually familiar with such a phenomenon in the dual picture where gravity is turned on and spacetime is curved, in which case momentum space is represented by covector fields and the metric induced on each fiber is dependent on the spacetime point. It can be said that relative locality is a dual gravity.
}

However, when we go to zero momentum we can no longer neglect the limitations on local measurements coming from the uncertainty principle of quantum mechanics. Quantum mechanically a particle of energy $p_{0}$ can only be localized with accuracy not greater than $\hbar / p_{0}$, and this combines with the relativity of locality expressed by 31 to give uncertainty relation of the form

$$
\Delta x \geq \frac{\hbar}{p_{0}}+|x||\Gamma| p_{0},
$$

characterizing the limitation on the sharpness of coordinates of particles with energy $p_{0}$. Taking small $p_{0}$ helps reduce the relative-locality features but increases the quantum mechanical uncertainty.

To understand the implications of this in more detail we will next specialize from the general case by imposing physical conditions which restrict the geometry of momentum space.

\section{SPECIALIZING THE GEOMETRY}

As we have seen, the geometry of momentum space can code several kinds of deformations of the energy-momentum conservation laws, which take advantage of the flexibility to choose the metric, torsion, curvature and non-metricity of the connection. This gives us an arena within which we can formulate and test new physical principles. These impose constraints on the choice of the geometry of $\mathcal{P}$. To illustrate this we next turn from the general case to show how a set of simple principles restricts us to a one parameter set of momentum space geometries, and consequently, an almost unique set of experimental predictions.

Consider the following four increasingly strong physical principles:

1. The correspondence principle: Special relativity describes accurately all processes involving momenta small compared to some mass scale $m_{c}$. While it is natural to presume that $m_{c} \approx m_{p}$, the scale $m_{c}$ should be determined experimentally.

2. The weak dual equivalence principle: The algebra of combination of momenta, and hence the geometry of $\mathcal{P}$ are universal; they do not depend on which kinds of particles are involved in interactions.

3. The strong dual equivalence principle or $E=m c^{2}$ : There is equivalence between the rest mass energy defined by the metric and the inertial mass which involves the connection.

4. Maximal symmetry. The geometry of momentum space is isotropic and homogeneous: invariant under Lorentz transformations and invariant under "translations".

The geometry of momentum space is discussed in more detail in [2] where it is precisely shown how these principles lead to a unique geometry. This unique geometry is characterized by a constant: the dual cosmological constant which as 
the dimension of an inverse mass square. When this dual cosmological constant is zero we recover usual special relativity, when it is non zero, momentum space is genuinely curved.

The first principle implies first that the metric of momentum space is a Lorentzian metric ( which we already have implicitly assumed). It also implies that the torsion and nonmetricity of $\Gamma_{a}^{b c}$ must be at least of order $1 / m_{p}$, while curvature must be of order of $1 / m_{p}^{2}$.

The weak equivalence principle implies that the combination of momenta do not depend on the colors or charges of particles and is the same as the composition for identical particles. For identical particles there is no operational way to give an order of the combination rule if we have Bose statistics, therefore taken strongly, that is if we do not allow for any modification of the statistics of identical particles, this principle also implies that the product is symmetric and hence the connection is torsionless.

The strong equivalence principle relates the metric and the connection of $\mathcal{P}$ by imposing that the connection is metric compatible. The metric determines distance between two points in momentum space, and hence governs the mass shell relations of single particles, while the?connection determine what is the straightest path between two points, and?hence is determined by interactions which combine momenta. Since they are given by different physics, they are in principle independent. However, there are indications, to be discussed in [3] that, at least in some cases, the non-metricity of the connection is related to violations of the equivalence between relativistic energy and mass. It is intriguing that Einstein's observation that in a relativistic theory $E=m c^{2}$ appears to relate the metric and the connection of momentum space.

The first three principles impose therefore that the geometry of momentum space is entirely fixed by a Lorentzian metric. The connection is then the unique connection which is torsionless and compatible with the metric.

The fourth principle of maximal symmetry is the most restrictive. This could be called the principle of "special relative locality" since it essentially implies a unique fixed dual geometry on momentum space. What it means in a nutshell is that the space of Killing vector fields of the metric form a ten dimensional Lie algebra. This symmetry algebra also preserve the connection. This implies that there exists Lorentz transformations $\Lambda$ acting on $\mathscr{P}$ fixing the identity 0 such that

$$
\Lambda(p \oplus q)=\Lambda(p) \oplus \Lambda(q) .
$$

This implies that the conservation law transform covariantly under Lorentz transformations

$$
\mathcal{K}(\Lambda k)=\Lambda \mathcal{K}(k)
$$

Hence, if we impose that the geometry of $\mathcal{P}$ is invariant under Lorentz transformations then we gain an action of the Lorentz group on the phase space $\Gamma$. From this we can conclude that there are for each interaction event, a family of local observers which see the interaction to take place at $z^{a}=0$ and hence be local.
There also exist a notion of translations ${ }^{6} T_{r}$ such that $T_{r}(0)=r$ and

$$
T_{r}\left(p \oplus_{k} q\right)=T_{r}(p) \oplus_{T_{r} k} T_{r}(q)
$$

where $\oplus_{k}$ is the translated combination rule (12).

Here we expand more on how Lorentz invariance can be made compatible with curved momentum space. First it is well known that around the identity 0 we can set up a special set of coordinates: The Riemannian normal coordinates. In this coordinates the distance from 0 is given by the usual flat space formula hence the mass shell condition in this coordinates is simply ${ }^{7}$

$$
C(k)=k_{0}^{2}-k_{i}^{2}-m^{2}=0 .
$$

The Lorentz transformations that preserves the zero momenta and the metric therefore acts in the usual manner in this coordinate systems.

Moreover under the hypothesis of homogeneity these coordinates can be extended to cover almost all the manifold $P$. The Lorentz generators therefore satisfy the usual algebra. If we assume in turn that the Lorentz transformations are canonical transformations preserving the Poisson bracket, they also satisfy the usual Poisson algebra. That is given the boost and rotation generators $N_{i}$ and $M_{j}, i, j, k=1,2,3$, we have

$$
\left\{M_{i}, M_{j}\right\}=\varepsilon_{i j k} M_{k}, \quad\left\{M_{i}, N_{j}\right\}=\varepsilon_{i j k} N_{k}, \quad\left\{N_{i}, N_{j}\right\}=-\varepsilon_{i j k} N_{k}
$$

and the generators act on the momentum space $\mathcal{P}$ through these brackets. Moreover, as in special relativity, we assume that the momentum space $\mathcal{P}$ splits into collections of orbits of the Lorentz group: the zero momentum point, which is left invariant by Lorentz transformations; the positive and negative energy mass shells of massive particles; the light cone corresponding to massless particles; and mass shells of tachyons of imaginary mass. It is a direct consequence of this assumption that the function $C(k)$ in the mass shell condition 19 ) must be a Lorentz scalar, so that all Lorentz observers agree what the value of the invariant mass $m^{2}$ is. It follows then that vectors corresponding to the infinitesimal Lorentz transformations are Killing vectors of the metric (4), so that the surfaces of constant distance from the origin (the point in $\mathcal{P}$ corresponding to zero momentum) are orbits of action of Lorentz group.

Combining the assumptions of Lorentz symmetry with translation invariance or, equivalently, homogeneity then completely determines the geometry of $\mathcal{P}$, up to an overall scale. Indeed, according to the latter the geometry of a fixed mass orbit $|k|^{2}+m^{2}=0$ is the same of all the masses. Thus the geometry of $\mathcal{P}$ is not only invariant under Lorentz transformations acting along the orbits, but also under translations,

\footnotetext{
6 The algebra of translations on momentum space does not have to be commutative. It can be defined to be the left translation $T_{r}(p) \equiv r \oplus p$.

${ }^{7}$ The distance $D^{2}\left(k, k^{\prime}\right)$ of two points away from the origin do not assume this simple form 44, which applies only to measurements of distances from the origin. Consequently, the action of lorentz boosts on the geometry can be non-trivial even if the action on the coordinates $k_{a}$ in which 44. holds is linear.
} 
mapping one orbit to another. All together we have therefore the 10-parameter (in 4 dimensions) group of transformations that leave the geometry of the momentum space invariant, and therefore this space is a maximally symmetric manifold. It is well known that there are only three such manifolds: the flat space and the (anti-) de Sitter spaces. On all three of these spaces the Lorentz group action is naturally defined. Notice that the $\mathcal{P}$ may be a submanifold of one of these spaces, satisfying the requirements that all the Lorentz orbit belong to this submanifold.

Two points are worth noting as we now transition from discussing the theoretical frameworks to a first sketch of their phenomenological implications. The two cases of positive and negative constant curvature on $P$ are expected to be rather different in their phenomenological implications. Also, while action of the lorentz transformations will be induced on the spacetime coordinates, this action may be deformed and will depend on which coordinates are used to label spacetime events and processes. As we have seen the induced spacetime coordinates are observer dependent, and are also either non-commutative or energy-momentum dependent. The details of how these affect the action of lorent transformations on spacetime reserved for discussion in a future publication.

\section{DETERMINING EXPERIMENTALLY THE GEOMETRY OF MOMENTUM SPACE}

In the nineteenth century, Gauss proposed that the geometry of space should be empirically determined, and there is a legend he set about measuring the curvature of space by determining the angles between three mountain peaks. Similarly, we here propose that the geometry of momentum space is to be measured rather than assumed. In this section we establish that the geometry of momentum space can produce observable effects. We do this by means of simple sketches of idealized experiments. We postpone for further work the consideration of observations that could actually be done with present and near future technology.

There are two classes of experiments that can be contemplated. There are experiments which aim to measure features of the geometry of momentum space; these can be distinguished from tests of the specific hypotheses described in the previous section. We describe two examples from the first class, which aim to measure the curvature of momentum space.

The first example we have chosen is from atomic physics, and shows that the curvature of a connection on momentum space, defined by (11) corresponds to a measurable quantity. We assume the availability of a beam of atoms of same type prepared all in the ground state with the same initial momentum. The idealized measurement procedure we consider assumes the availability of four energy levels: in addition to the ground state it involves excited levels $I, I I$ and $I I I$. Ideally we would want these energy levels to be such that there is a small step in energy from ground to I excited, a large step in energy from $I$ to $I I$ and another small step in energy from $I I$ to III. We excite the atoms with lasers tuned to the transition between these states as shown in Figure 2.

(a)
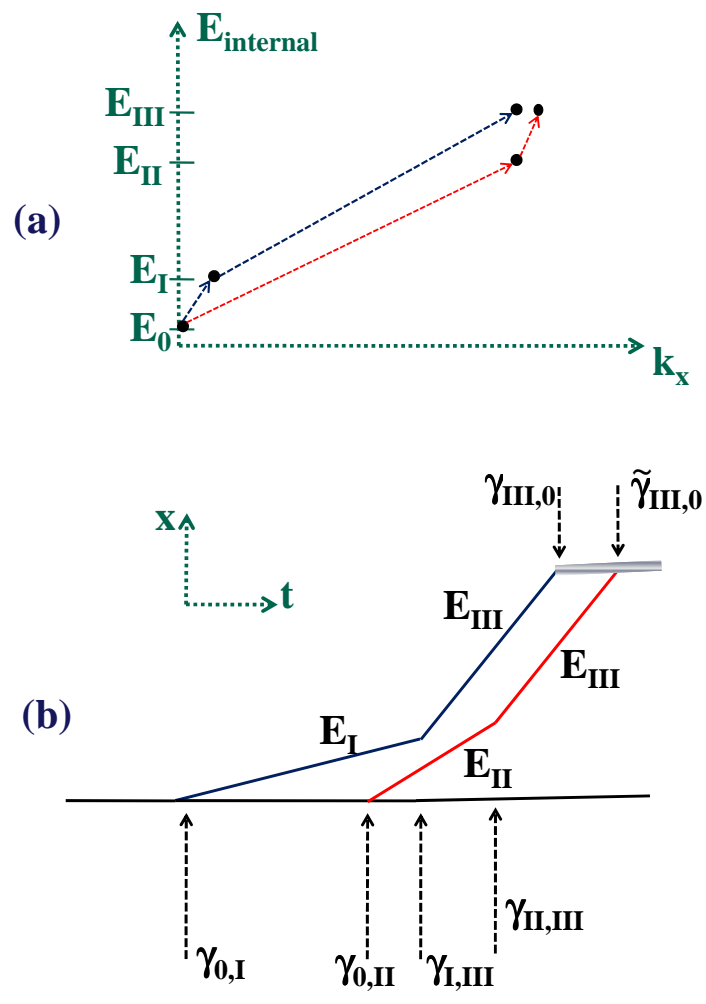

FIG. 2: Schematic description of a measurement procedure aimed at exposing the implications of a non-trivial connection on momentum space. The routeA and routeB described in the text are here shown both in momentum space (panel (a)) and as spacetime trajectories of the atoms (panel (b)).

By the scheme in Figure 2 we can bring atoms from the ground state to the level III excited following two routes:

$$
\text { routeA } \equiv \text { ground } \rightarrow I \rightarrow I I I
$$

and

$$
\text { route } B \equiv \text { ground } \rightarrow I I \rightarrow I I I
$$

The resulting momenta for the two processes are $\left(p \oplus k_{0, I}\right) \oplus$ $k_{I, I I I}$ and $\left(p \oplus k_{0, I I}\right) \oplus k_{I I, I I I}$, and can be compared by measuring the laser frequency needed to bring the route $A$ atoms back to the ground state and the laser frequency needed to bring the route $B$ atoms back to the ground state. In the idealized situation of $k_{0, I} \simeq k_{I I, I I I} \equiv q, k_{0, I I} \simeq k_{I, I I I} \equiv Q$, this procedure would give a clean comparison between $(p \oplus q) \oplus Q$ and $(p \oplus Q) \oplus q$, which following the analysis we offered in the previous sections is indeed a sensitive indicator of the nontriviality of the connection on momentum space. We expect 
that other more practical and sensitive measurement procedures for the geometry of momentum space will be gradually found, if a dedicated research effort is inspired by our proposal. The simplicity of the scheme described in Figure 2 serves the purpose of showing very clearly that the geometry of momentum space can manifest itself in measurable quantities. The procedure sketched in Figure 2 is also representative of a whole class of strategies for measuring non-associativity and/or non-commutativity of the law of composition of momenta, which, as we have shown, are expressed respectively by the curvature and torsion of a connection on $\mathcal{P}$.

It is interesting to note also that there is a simple analogy between the non-linear composition of momenta we have discussed here and the nonlinear law of composition of velocities, in Special Relativity. It is not always stressed that the composition law of velocities in special relativity is nonassociative [5]. This non-associativity is absent for addition of co-linear velocities but is measured in Thomas procession [6]. This suggest an experiment inspired by the Thomas precession experiment [7]. The idea is to follow a system in orbit (an electron in an atom or a particle circling in the LHC). Such a system is enclosing a loop in momentum space at each period of revolution, which enclose the curvature in momentum space. At each period the localization of the orbiting particle will be shifted compared to the localization of a particle at rest. Effectively, the particle will experience an infinitesimal boost $N_{i}$ at each period given by

$$
N_{i}=\frac{\Delta A_{c d}}{m_{P}^{2}} R^{c d a}{ }_{i} p_{a} \approx \frac{\Delta A_{c d}}{m_{P}^{2}} m R^{c d 0}{ }_{i}
$$

where $\Delta A_{c d}$ is the area of the loop in momentum space and $m$ the mass of the particle. One should be able to observe then a spacetime displacement due to relative locality effect. Even if this effect is tiny this type of observation possess a huge potential since it is a cumulative effect and we can use the large number of orbits that develop over time.

These experiments represent ways to measure the curvature of momentum space, which is the main effect that needs to be probed. We can also test more broadly the solidity of the strong and weak dual equivalence principle. The metricity of the connection could be tested by looking for violations of the equivalence between mass and rest energy. There are numerous nuclear physics experiments that rely on the equivalence between mass and energy, the accuracy that is achieved in these experiments is not Planckian, but they clearly deserve a closer scrutiny in light of the new principle we propose.

It will be also important to have a direct experimental bound on the momentum space torsion. One way to probe it is to put Bose statistics under experimental scrutiny, since we have argued that a non vanishing torsion will in effect correspond to a modification of the statistics ${ }^{8}$. This issue deserves

\footnotetext{
${ }^{8}$ The usual argument in favor of the standard statistics uses crucially the existence of an absolute spacetime, and the independence of the state of a system on its momentum space history. Logically, this derivation should be revisited in light of the relative locality principle
}

a deeper analysis in order to propose specific effects to look for. Also one could imagine a momentum space Eötvos experiment showing that all type of matter add momentum in the same way.

We can add that a non-trivial geometry of momentum space can in the most general form produce deformations of well tested symmetries of quantum field theory including $C P T$ and crossing symmetry [4]. This is because they imply nonlinearities in conservation laws, which would show up as violations of the linear form of those laws. For example, we expect that standard arguments on crossing symmetry in which an incoming particle with four momentum $k_{a}$ is replaced by an outgoing antiparticle with four momentum $-k_{a}$ will be deformed so the antiparticle has instead momentum $\ominus k_{a}$. This will introduce non-linearities which will show up as violations of the usual crossing symmetry. Thus the tight experimental constraints on these symmetries constrains the geometry of momentum space. This is expressed by the correspondence principle we discussed in the previous section. Thus, a first task for phenomenologists will be to understand the bounds on the mass scale $m_{c}$ from existing tests of fundamental symmetries in quantum field theory [4].

These examples illustrates that the principles proposed here open a new type of investigation, both experimental and theoretical, into the geometry of momentum space.

\section{CONCLUSIONS}

The passage from special relativistic locality to relative locality reminds us of the passage we navigated a century ago from absolute space to spacetime. The additivity of velocity implies there is an absolute time by which velocity is measured. If we hypothesize that the combinations of velocity might become non-linear, without weakening the principle of the relativity of inertial frames, we need an invariant scale, to measure the scale of the non-linearities, which must be a velocity itself. Hence there is an invariant velocity we call $c$. This then allows us to interchange distances and times, which makes possible the existence of an absolute spacetime, which replaces the notion of absolute space. Space itself remains, but as an observer dependent concept, because of the relativity of the simultaneity of distant events.

Similarly, as we observed above, the additivity of momenta and energy implies the existence of an absolute spacetime. When we contemplate weakening that to a non-linear combination rule for momenta in physical interactions, we need an invariant momentum scale. We have taken this scale to be $m_{p}$ but of course from a phenomenological point of view it should be taken as having a free value to be constrained by experiment. This, together with $\hbar$ makes it possible to interchange distances and momenta, which makes possible the mixing of spacetime coordinates with energy and momenta, so that the only invariant structure is the phase space. We saw above explicitly how non-linearity in conservation of energy and momentum directly forces translations of spacetime coordinates to depend on momenta and energies. Local spacetime remains, but as an observer dependent concept, because of the 
relativity of the locality of distant events.

Relative locality suggests novel points of departure for attempts to discover the right quantum theory of gravity. Here, we have discussed its implications in a semiphenomenological perspective framed by the approximation (1) but it is possible it goes deeper. For example, one can suggest that the fundamental description is one in which there is dynamical curvature in phase space, so that the fundamental constants are $G_{N e w t o n}$ and $m_{p}$. This implies that Planck's constant could be a derived quantity, $\hbar=G_{\text {Newton }} m_{p}^{2}$, suggesting that quantum mechanics is emergent from a dynamics of phase space geometry.

The idea that momentum space might be curved has a long history. To our knowledge, it was first put forward more than 70 years ago in a paper by Max Born [8]. The idea seems to have been next discussed independently in the celebrated paper by Snyder [9], which was followed by series of papers of Russian physicists from the 1950s to the 1980s [10]. There the main motivation was the ultraviolet divergencies in quantum field theories; it was hoped that curving momentum space may help taming these divergencies.

As Snyder first pointed out, the curvature of momentum space implies the non-commutativity of spacetime coordinates which generate translations on momentum space. A picture of quantum geometry in which a curved momentum space is dual to a non-commutative spacetime was introduced by Majid, who stressed the relation to quantum groups [11]. This idea was realized in the construction of $\kappa$-Minkowski spacetime which is a non-commutative geometry invariant under a deformation of Poincare symmetry known as the $\kappa$-Poincare algebra [12], [13] ${ }^{9}$.

Still more generally, Alain Connes has stressed [14] that the fundamental observables are spectra of energy and momentum, tightly cementing the relationship between the geometry of momentum space and non-commutative geometry.

Another motivation comes from lower dimensional physics. It is by now well established by different methods [15.-22] that in $2+1$ dimensions, where gravity is described by a topological field theory [23], the effective momentum space of particles is deformed and becomes a (curved) three dimensional group manifold.

So the world we have described here is realized in at least one well developed example, which is quantum gravity coupled to matter in $2+1$ dimensions.

In the physical $3+1$ dimensions the situation is less clear. It has been suggested that, as in the 3D case, the "no gravity" limit is governed by topological field theory, effectively leading to curved momentum space [24]. The curved momentum space was also employed in the context of group field theory, which generates amplitudes for spin foam models of quantum gravity [25]. Quantum field theory with curved momentum space has been recently discussed in detail in [26]. The dynamical momentum space in the context of the cosmological

\footnotetext{
${ }^{9}$ The geometry of $\kappa$-Poincare theory fits the general scheme presented here with a connection with non-zero torsion but zero curvature.
}

constant problem has been recently discussed [27].

Last but not least, momentum spaces of constant curvature find its natural application as a model of Doubly (or deformed) Special Relativity (DSR) [28, 30, 32], whose second observer independent scale is naturally associated with the curvature of the momentum space [30] ${ }^{10}$ (see also [31].) These formulations were closely related to non-commutative geometry. A second formulation of DSR expressed the same idea as an energy dependence of spacetime [32]. For a long time it has been suspected that these were different ways of formulating the same theories, the developments of this paper show how an energy dependent metric and non-commutative spacetime coordinates are different ways of expressing a deeper idea, which is relative locality.

The non-associativity of momentum addition in noncommutative geometry was also explored in [5], which stressed the analogy to the non-associativity of velocity composition in special relativity. The non-associativity of momentum conservation was also further explored in [33] ${ }^{11}$.

The issue of possible macroscopic non-local effects in DSR was raised several times [34-38]. Attempts to address these issues led to some partial anticipations of the ideas proposed here [39-41]. From the present perspective we can appreciate that the concern was well justified and there is a pot of gold under the rainbow of apparent non-locality. At the same time, the principle of relative locality tells us that the theories discussed here do not contain physical non-localities of the kind that were suggested in [35.37]. Instead, energy-momentum conserving interactions are always local in spacetime when observed by observers who are local to them. The fact that a distant observer is only able to localize an event involving several particles with different momenta to within a region whose scale is proportional to its distance is inevitable in theories with curved momentum spaces, and should not be misconstrued as entailing a violation of the physical principle that physical interactions are local.

Nonetheless, if theories are still local, in the restricted sense of relative locality, there are, as we discussed above, new phenomena that can be studied experimentally. In addition, as will be explained elsewhere, the problem of whether photons emitted simultaneously are detected simultaneously, after traveling for very long distances, can be cleanly addressed. This is relevant for the timing of arrival time measurements in gamma ray bursts [43-45].

We have seen here that the notion of curved momentum space has generic and vivid consequences for our understanding of basic physics. We do not live in spacetime. We live in Hilbert space, and the classical approximation to that is that we live in phase space. If we think that we can filter out colors and frequencies of particles to arrive at a picture of particles

\footnotetext{
10 The existence of a regime defined by eq. 11 was discussed by several authors including 42, 47 48].

11 While finishing this paper we learned that our construction is related to the mathematical theory of loops developed, among others, by M. Kikkawa and L.V. Sabinin, [46]. We thank F. Girelli and E. Livine for pointing this out to us.
} 
moving in spacetime which is independent of the momenta those particles carry, that is only an illusion that has been possible because of the smallness of elementary particle scales in Planck units. Similarly, if we think that observers distant from each other see the same spacetime that is only because we are only beginning to make precise measurements of quantities where the combination $|z||\Gamma| k \mid / \hbar$ is not negligible.

The crucial idea underlying and unifying all these developments turns out to be one that is a direct consequence of the curvature of momentum space: the relativity of locality.

Even apart from fundamental physics, there are situations in condensed matter physics, where it is convenient to understand excitations as living in a curved momentum space[49]. The considerations of this paper may be relevant for those cases. Or, to put it the other way, just as some condensed matter or fluid systems provide analogues for relativity and gravity, it may be that condensed matter systems with curved momentum spaces may give us analogues to the physics of relative locality.

So look around. You see colors and angles, i.e. you are seeing into phase space. The idea that underlying it is an energy independent, invariant spacetime geometry could be an approximation, reliable only to the extent that we measure the geometry with quanta small compared to the Planck energy and we neglect phenomena of order of $|z||\Gamma| k \mid / \hbar$. Whether this is correct or not is for experimental physics to decide. If it turns out to be correct, then a new arena opens up for experimental physics and astronomy, which is the measurement of the geometry of momentum space.

\section{ACKNOWLEDGEMENTS}

We are very grateful to Stephon Alexander, Michele Arzano, James Bjorken, Florian Girelli, Sabine Hossenfelder, Viqar Husain, Etera Livine, Seth Major, Djorje Minic, Carlo Rovelli, Frederic Schuller, Guglielmo Tino, and William Unruh for conversations and encouragement. GAC and JKG thank Perimeter Institute for hospitality during their visits in September 2010, when the main idea of this paper was conceived. The work of G. Amelino-Camelia was supported in part by grant RFP2-08-02 from The Foundational Questions Institute (fqxi.org). The work of J. Kowalski-Glikman was supported in part by grants 182/N-QGG/2008/0 and N 202331139. Research at Perimeter Institute for Theoretical Physics is supported in part by the Government of Canada through NSERC and by the Province of Ontario through MRI.
[1] A. Einstein, Zur Elektrodynamik bewegter Körper, Annalen der Physik 17, 891 (1905) English translation On the electrodynamics of moving bodies, in The principle of relativity: a collection of original memoirs By Hendrik Antoon Lorentz, Albert Einstein, H. Minkowski, Hermann Weyl, Dover books. (English translation also at http://www.fourmilab.ch/etexts/einstein/specrel/specrel.pdf).

[2] L. Freidel, The geometry of momentum space, preprint in preparation.

[3] L. Smolin et al, preprint in preparation.

[4] G. Amelino-Camelia et. al, preprint in preparation.

[5] F. Girelli and E. R. Livine, Special Relativity as a non commutative geometry: Lessons for Deformed Special Relativity, Phys. Rev. D 81 (2010) 085041 [arXiv:gr-qc/0407098].

[6] L. H. Thomas, The motion of a spinning electron, Nature 117, 514 (1926); ibid. The Kinematics Of An Electron With An Axis, Phil. Mag. 3, 1 (1927). E. P. Wigner, On Unitary Representations Of The Inhomogeneous Lorentz Group, Annals Math. 40, 149 (1939) [Nucl. Phys. Proc. Suppl. 6, 9 (1989)].

[7] F. Girelli, E.R. Livine, Physics of Deformed Special Relativity: Principle of Relativity revisited, gr- qc/0412004; Deformed Special Relativity: problems and solutions, Braz. J. Phys. 35 (2005) 432- 438, gr-qc/0412079

[8] Max Born, A Suggestion for Unifying Quantum Theory and Relativity, Proc. R. Soc. Lond. A, 1938165 291-303.

[9] H. S. Snyder,Quantized space-time, Phys. Rev. 71, 38 (1947).

[10] Yu. A. Gol'fand, On the introduction of an "elementary length" in the relativistic theory of elementary particle, JETP 37 (1959) 504-509; English in: Soviet Physics JETP 10 (1960) 356360; V. G. Kadyshevskii, On the theory of quantization of space-time, JETP 41 (1961) 1885-1894, English in: Soviet Physics JETP 14 (1962) 1340-1346; Yu. A. Gol'fand, Quantum field theory in constant curvature p-spac, JETP 43 (1962)
256-257, English in: Soviet Physics JETP 16 (1963) 184-191; V. G. Kadyshevsky, M. D. Mateev, R. M. Mir-Kasimov and I. P. Volobuev, Equations Of Motion For The Scalar And The Spinor Fields In Four-Dimensional Noneuclidean Momentum Space, Theor. Math. Phys. 40 (1979) 800 [Teor. Mat. Fiz. 40 (1979) 363]; V. G. Kadyshevsky and M. D. Mateev, Quantum Field Theory And A New Universal High-Energy Scale: The Scalar Model, Nuovo Cim. A 87 (1985) 324.

[11] S. Majid, Meaning of noncommutative geometry and the Planck-scale quantum group, Lect. Notes Phys. 541 (2000) 227 [arXiv:hep-th/0006166].

[12] J. Lukierski, H. Ruegg, A. Nowicki et al., $Q$ deformation of Poincare algebra, Phys. Lett. B264 (1991) 331-338.

[13] S. Majid, H. Ruegg, Bicrossproduct structure of kappa Poincare group and noncommutative geometry, Phys. Lett. B334 (1994) 348-354. [hep-th/9405107].

[14] A. Connes, Non-commutative geometry Academic Press, 1994.

[15] H. -J. Matschull, M. Welling, Quantum mechanics of a point particle in $(2+1)$-dimensional gravity, Class. Quant. Grav. 15 (1998) 2981-3030. |gr-qc/9708054|.

[16] F. A. Bais, N. M. Muller, Topological field theory and the quantum double of SU(2), Nucl. Phys. B530 (1998) 349-400. |hepth/9804130].

[17] F. A. Bais, N. M. Muller, B. J. Schroers, Quantum group symmetry and particle scattering in $(2+1)$-dimensional quantum gravity, Nucl. Phys. B640 (2002) 3-45. [hep-th/0205021].

[18] C. Meusburger and B. J. Schroers, Poisson structure and symmetry in the Chern-Simons formulation of $(2+1)$-dimensional gravity, Class. Quant. Grav. 20 (2003) 2193 |arXiv:gr$\mathrm{qc} / 0301108$ ].

[19] G. Amelino-Camelia, L. Smolin and A. Starodubtsev, Quantum symmetry, the cosmological constant and Planck scale phenomenology, Class. Quant. Grav. 21 (2004) 3095 arXiv:hep- 
th/0306134|.

[20] L. Freidel, J. Kowalski-Glikman and L. Smolin, 2+1 gravity and doubly special relativity, Phys. Rev. D 69 (2004) 044001 [arXiv:hep-th/0307085].

[21] L. Freidel, E. R. Livine, Ponzano-Regge model revisited III: Feynman diagrams and effective field theory, Class. Quant. Grav. 23, 2021-2062 (2006). |hep-th/0502106].

L. Freidel and E. R. Livine, Effective $3 d$ quantum gravity and non-commutative quantum field theory, Phys. Rev. Lett. 96 (2006) 221301 |arXiv:hep-th/0512113].

[22] B. J. Schroers, Lessons from (2+1)-dimensional quantum gravity, PoS QG-PH (2007) 035 |arXiv:0710.5844 [gr-qc]].

[23] E. Witten, (2+1)-Dimensional Gravity as an Exactly Soluble System, Nucl. Phys. B 311 (1988) 46.

[24] J. Kowalski-Glikman and A. Starodubtsev, Effective particle kinematics from Quantum Gravity, Phys. Rev. D 78 (2008) 084039 [arXiv:0808.2613 [gr-qc]].

[25] L. Freidel, Group field theory: An Overview, Int. J. Theor. Phys. 44, 1769-1783 (2005). |hep-th/0505016|.

D. Oriti, The group field theory approach to quantum gravity: some recent results, arXiv:0912.2441 [hep-th].

[26] M. Arzano, Anatomy of a deformed symmetry: field quantization on curved momentum space, arXiv:1009.1097 [hep-th].

[27] L. N. Chang, D. Minic, T. Takeuchi, Quantum Gravity, Dynamical Energy-Momentum Space and Vacuum Energy, Mod. Phys. Lett. A25 (2010) 2947-2954. [arXiv:1004.4220 [hep-th]].

[28] G. Amelino-Camelia, Testable scenario for relativity with minimum-length, Phys. Lett. B 510, 255 (2001) |arXiv:hepth/0012238].

[29] G. Amelino-Camelia, Relativity in space-times with shortdistance structure governed by an observer-independent (Planckian) length scale, Int. J. Mod. Phys. D 11, 35 (2002) |arXiv:gr-qc/0012051].

[30] J. Kowalski-Glikman, De Sitter space as an arena for doubly special relativity, Phys. Lett. B 547 (2002) 291 |arXiv:hepth/0207279].

[31] J. W. Moffat, "Quantum gravity momentum representation and maximum invariant energy," arXiv:gr-qc/0401117

[32] J. Magueijo, L. Smolin, Lorentz invariance with an invariant energy scale Phys. Rev. Lett. 88 (2002) 190403. |hepth/0112090]; Generalized Lorentz invariance with an invariant energy scale, gr-qc/0207085. Phys.Rev. D67 (2003) 044017.

[33] F. Girelli, Snyder Space-Time: K-Loop and Lie Triple System, SIGMA 6, 074 (2010). [arXiv:1009.4762 [math-ph]].

[34] G. Amelino-Camelia, Doubly special relativity: First results and key open problems Int. J. Mod. Phys. D11 (2002) 1643
[arXiv:gr-qc/0210063]. (See the paragraph before eq. 21).

[35] S. DeDeo and C. Prescod-Weinstein, Energy-Dependent Speeds of Light for Cosmic-Ray Observatories arXiv:0811.1999

[36] R. Schutzhold, W. G. Unruh, Large-scale nonlocality in 'doubly special relativity' with an energy-dependent speed of light, JETP Lett. 78 (2003) 431-435. [gr-qc/0308049].

[37] S. Hossenfelder, Bounds on an energy-dependent and observerindependent speed of light from violations of locality, Phys. Rev. Lett. 104 (2010) 140402. |arXiv:1004.0418 [hep-ph]].

[38] M. Arzano, Comment on 'Large scale nonlocality in 'doubly special relativity' with an energy dependent speed of light', Igrqc/0309077].

[39] G. Amelino-Camelia, M. Matassa, F. Mercati and G. Rosati, Taming nonlocality in theories with deformed Poincare symmetry, arXiv:1006.2126 [gr-qc]; U. Jacob, F. Mercati, G. AmelinoCamelia and T. Piran, Modifications to Lorentz invariant dispersion in relatively boosted frames, Phys. Rev. D 82 (2010) 084021 [arXiv:1004.0575 [astro-ph.HE]]

[40] L. Smolin, On limitations of the extent of inertial frames in noncommutative relativistic spacetimes, arXiv:1007.0718, Classical paradoxes of locality and their possible quantum resolutions in deformed special relativity, arXiv:1004.0664

[41] M. Arzano, J. Kowalski-Glikman Kinematics of a relativistic particle with de Sitter momentum space, arXiv:1008.2962 [hepth].

[42] J. Kowalski-Glikman, S. Nowak, Doubly special relativity and de Sitter space, Class. Quant. Grav. 20 (2003) 4799-4816. |hepth/0304101].

[43] John Ellis, N.E. Mavromatos, D.V. Nanopoulos, Probing a Possible Vacuum Refractive Index with Gamma-Ray Telescopes Phys. Lett. B674 (2009) 83. |arXiv:0901.4052]

[44] G. Amelino-Camelia and L. Smolin, Prospects for constraining quantum gravity dispersion with near term observations, Phys. Rev. D 80 (2009) 084017 [arXiv:0906.3731 [astro-ph.HE]].

[45] A. A. Abdo et al, A limit on the variation of the speed of light arising from quantum gravity effects Nature 462 (2009) 331.

[46] Kikkawa M., Geometry of homogeneous Lie loops, Hiroshima Math. J. 5 (1975), 141-179; Sabinin L.V., Smooth quasigroups and loops, Kluwer Academic Publishers, Dordrecht, 1999

[47] S. Majid and R. Oeckl, Twisting of quantum differentials and the Planck scale Hopf algebra, Commun. Math. Phys. 205 (1999) 617 [arXiv:math/9811054].

[48] C. Rovelli, A note on DSR, arXiv:0808.3505 [gr-qc].

[49] Shunji Matsuura, Shinsei Ryu, Momentum space metric, nonlocal operator, and topological insulators, arXiv:1007.2200. 